\title{
Performance Analysis of De-registration Strategies in Personal Communication Network
}

\author{
Rajeev R. Kumar Tripathi \\ SSSIST, Sehore (MP), India
}

\author{
Sudhir Agrawal \\ BIT, GIDA (UP), India
}

\author{
Swati Tiwari \\ ITW, GIDA (UP), India
}

\begin{abstract}
When a mobile terminal (MT) leaves its registration area (RA) and enters into new RA, the serving Visitor Location Register (VLR) sends a registration request to the Home Location Register (HLR).On receiving this request the HLR sends a de-registration request to the old VLR (which was serving the MT).After deregistering the MT, the old VLR acknowled ges the request of HLR and then the HLR confirms the registration of the MT by sending the acknowledgement message to the new VLR. This approach is called explicit deregistration scheme. Implicit de-registration is a variant scheme, in which we can save the cost by ignoring the explicit de-registration message to the old VLR and its acknowled gement to the HLR. In terms of cost, implicit deregistration strategy is efficient than the explicit as we are saving de-registration signal exchange cost. However, at old VLR we may have the invalid entries. These invalid entries increase the database size at the VLR. To remove the invalid entries from the VLR, various de-registration schemes were suggested like polling, timeout and the group de-registration scheme. In this paper a comparative an aly sis is performed. On the basis of the cost incurred and the numerical result shows that the group de-registration scheme is efficient than the timeout and polling scheme.
\end{abstract}

\section{Keywords}

Explicit de-registration, Implicit de-registration, polling, timeout and group de-registration.

\section{INTRODUCTION}

In Personal Communication Network registration is a process by which the mobile terminal informs the network about its current location. In network mobility management protocols such as IS-41[8] and GSM [9] de-registration can be efficiently achieved as part of the registration process. When an MT leaves its RA and enters into the new RA belonging to the different VLR, the MT sends a registration message to the new VLR [1]. On the reception of this message the current VLR sends the registration request to the HLR [11]. In response the HLR sends a cancellation message to the old VLR to cancel the entry of the MT. The old VLR after cancellation sends an acknowledgement to the HLR and then HLR sends a confirmation message as an acknowled gement to the new VLR. In this way registration of new MT takes place. This scheme is called explicit de-registration scheme [2]. Explicit de-registration scheme is shown in the fig (1) [3]. In explicit de-registration scheme the HLR is sending a cancellation request to the old VLR and in the response old VLR is sending an acknowledgement to the HLR.

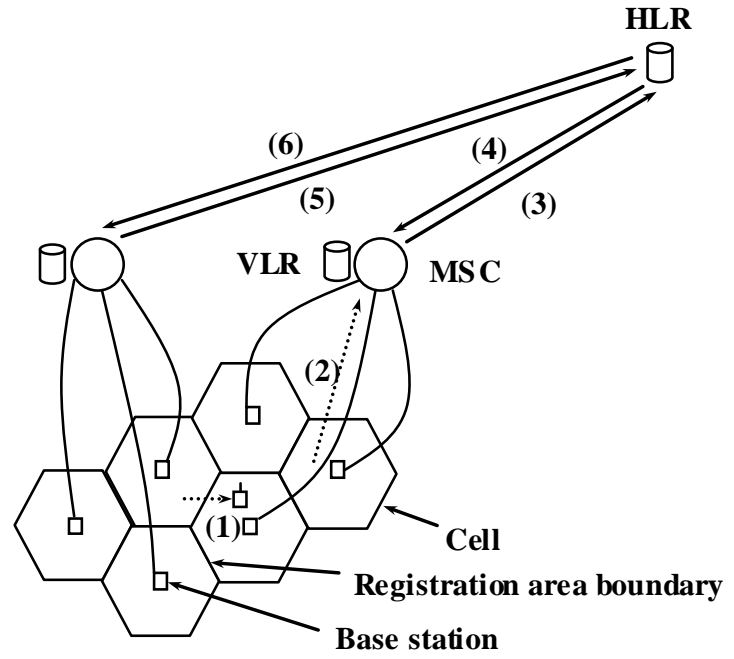

Fig 1: Explicit de-registration

This process is being done only to remove the entry of the MT from the old VLR because the MT is now in different RA and going to be served by the new VLR. Implicit de-registration $[4,5,7,10]$ is a novel technique which can be used to save signaling messages on the network. Whenever an MT moves from one RA to another within the same tier of service, it must register to the new VLR. Then the new VLR signals the arrival of the MT to the HLR, which updates the user location record. The HLR then deregisters the user from the old VLR. When a call arrives for the user, the HLR is queried and the call is routed to the new registration area. The de-registration process [12] to the old VLR is unnecessary from a call delivery point of view. It is only necessary to clean up old registration records and free up registration resources at the old VLR. The network de-registration signaling can be eliminated i.e. the MT can be implicitly deregistered at the HLR from the old VLR, provided that old VLR has a method of cleaning up old registration records. In implicit deregistration scheme we ignore the messages to the old VLR and hence we can save the cost consumed in the message transmission. So, implicit de-registration scheme is efficient than the explicit de-registration scheme. If an MT leaves the RA, the VLR should remove its invalid entry from the database. To remove the invalid entries from the old VLR side, we should have some methods by which the old VLR can determine whether the MT is currently under its RA or not. For this purpose some de-registration schemes were suggested as (1) polling de-registration scheme [5] (2) timeout de-registration scheme [4] and (3) group de-registration scheme [2]. In section II, these de-registration schemes are discussed. 


\section{DE-REGISTRATION SCHEMES 2.1 Polling De-registration Scheme}

Polling is a method of determining whether the mobile terminal is still present within the RA or not. An MT is periodically polled by receiving an alert in normal fashion i.e. as if the network has an incoming call to be delivered to the MT. In this scheme BSC belonging to the VLR transmit the polling signal through the BTSs. In response if MT is present in that RA, it sends the acknowledgment.

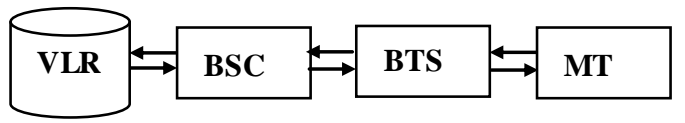

Fig (2) Polling de-registration

If VLR does not get the acknow led gement from an MT, it assumes that MT has moved from its RAs to another RA. Polling de-registration is shown in fig (2).

\subsection{Timeout De-registration Scheme}

It is a refinement of the above scheme. In this scheme the MT periodically sends registration request to the VLR. This is the way by which the VLR determines that the MT is currently under its RAs. If the VLR does not get the registration request from the MT periodically, it is assumed that the MT has moved from its RAs.

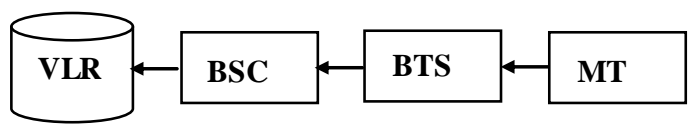

Fig (3) Timeout de-registration

The difference between timeout and polling is, in case of timeout MT alarms its presence in RA by sending signal periodically to VLR through BTS \& BSC but in case of polling, VLR sends polling signal with the help of BSC and BTS and receives acknowledgement from MT about its presence/absence in that RA. Timeout de-registration is shown in the fig (3).

\subsection{Group De-registration}

This scheme is more refinement in de-registration strategies. In this scheme HLR maintains a list of old MT list called old mobile list (OML) for each VLR. When an MT leaves one RA and enters into another RA belonging to the different VLR, new VLR sends registration request to the HLR for the MT. On receiving the request HLR puts the MT's identification into the OML of the old VLR and sends an acknowledgement along with the OML of new VLR. On reception of this acknowled gement the new VLR registers the MT and OML is used by the VLR to remove the entries of those MT who have left this VLR. This scheme can be described as follows:

1) When an MT enters into a new RA, the VLR associated with the new RA sends a registration request to the HLR.

2) The HLR on reception of the request updates the MT's service profile to point to the new RA. Instead of sending a registration cancellation message to the old VLR immed iately, the HLR keeps the identification of the MT in the OML.

3) The HLR sends a registration acknowled gement message to the new VLR of the MT along with a copy of the MT's service profile and all MT identifications kept in the OML of new RA, and empties this OML.

4) After receiving the registration acknowledgement message, the new VLR associated with the new RA picks up the move-out MT's identifications from the registration acknowledgement message, and removes these MTs service profiles from the VLR.

Thus the invalid MT's identifications are removed from the VLR every time a new MT enters the RA.

\section{PER FORMANCE ANALYSIS}

An analytical model to evaluate the performance of the conventional HLR/VLR architecture (using explicit deregistration strategy) has been presented in this paper and a comparison of the same is made with the modified HLR/VLRs (using different implicit de-registration strategies). In this analysis, a hierarchical tree of $\mathrm{R}$ layers is used, as shown in Fig. 4[6]. The layer $\mathrm{R}$ contains the root node and the layer 1 contains the leaf nodes. A database is installed on each node of the tree and the MTs are assigned to the leaf nodes. In the HLR/VLRs scheme, the network database, HLR, is situated on the only node of layer R and the VLRs are installed on the leaf nodes.

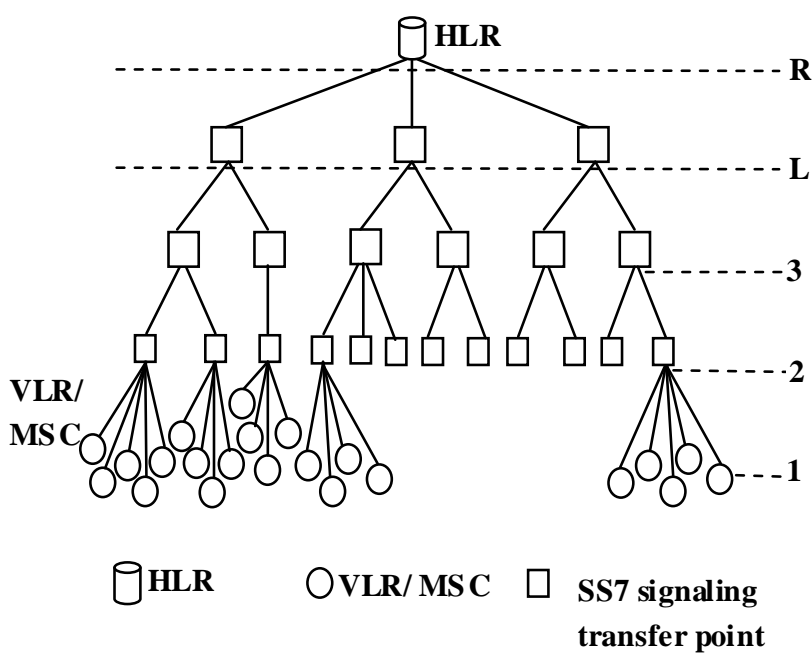

Fig. 4: HLR/VLR architecture

The following terms are used in performance analy sis:-

$\mathbf{m}_{\mathbf{x}, \mathbf{y}}$ Layer of the closest common node to RA $\mathrm{x}$ and RA $\mathrm{y}$.

p Probability that the MT move is intra-VLR.

q Probability that the called and the calling MTs are served by the same VLR.

n New RA of the MU.

a Old RA of the MU.

s RA of the calling unit (source).

d RA of the called MU (destination).

$\mathrm{P}\left(\mathbf{m}_{\mathbf{x}, \mathbf{y}}=\mathrm{i}\right)$ is defined as the probability that the closest common node to LA $x$ and LA $y$ is in layer $i$. This probability can be given by the following equation.

$\begin{aligned} P\left(m_{a, n}=i\right)= & p(1-p)^{i-1} \text { for } i=1,2 \ldots \ldots \ldots R-1 \\ & (1-p)^{i-1} \text { for } i=R \ldots \ldots \ldots \ldots \ldots . . .\end{aligned}$

$P\left(m_{s, d}=i\right)=q(1-q)^{i-1}$ for $i=1,2 \ldots \ldots . . R-1$ 


$$
(1-q)^{i-1} \text { for } i=R .
$$

We furthermore denote the costs of various operations used in this analy sis as follows:

$\mathbf{T}(i, j)$ : Cost of transmitting a message over a link between two adjacent layers $i$ and $j$.

$\mathbf{C}_{\mathbf{m}}$ (i): Cost of accessing or updating a database in layer i.

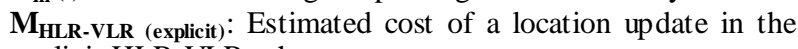
explicit HLR-VLR scheme.

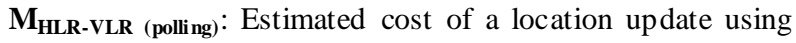
polling scheme.

M $_{\text {HLR-VLR (timeout) }}$ : Estimated cost of a location update using timeout scheme.

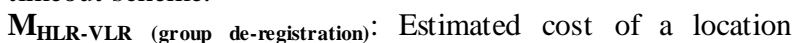
update using group de-registration scheme.

Estimated cost of location update in explicit HLR-VLR scheme is given as:

$$
\begin{aligned}
M_{H L R-V L R \text { (sxpicit) }} & =\left[P\left(m_{a, n^{-}} 1\right) \times C_{m_{k}}(1)\right] \\
& +\sum_{i=2}^{R} P\left(m_{a, n}-i\right) \\
& \times\left\{2 \times C_{m}(1)+C_{m}(R)\right. \\
& +4 T(1, R)\} \ldots \ldots(3)
\end{aligned}
$$

The first part of Eq. (3) is the cost of location update in intraVLR move. The second part illustrates the scenario after an inter-VLR move. $\mathrm{T}(1, \mathrm{~L})=\mathrm{T}(1,2)+\mathrm{T}(2,3)+\ldots \ldots . .+\mathrm{T}(\mathrm{L}-1$, $\mathrm{L}$ ) is equal to the cost of traversing links between a node of lay er 1(i.e., VLR) and the node of lay er R (i.e., where an HLR is located). This cost is multiplied by 4 because new VLR sends registration request to the HLR, the latter sends cancellation request to the old VLR, old VLR sends an acknowledgement in response to the HLR and finally HLR confirms the registration of new MT at the new VLR.

Transmission cost of the message is described as follows:

$\mathrm{T}(1, \mathrm{~L})=\mathrm{T}(1,2)+\mathrm{T}(2,3)+\ldots \ldots . .+\mathrm{T}(\mathrm{L}-1, \mathrm{~L})$

$\mathrm{T}(1,2)$ will give the result 2 ; $\mathrm{T}(2,3)$ will give the result 3 and so on.

In polling de-registration scheme periodical message transmission cost can be calculated as:

$\mathrm{T}(1,1)=1$.This is because; MT, BTS, BSC and VLR are collectively allocated in the same layer. Thus total cost at VLR end will be $2 * \mathrm{~T}(1,1)=2$ as link is being traversed twice. In the same way in timeout de-registration strategy message transmission cost will be 1 .Hence at this point we can now conclude that timeout de-registration scheme incurs less cost than polling.

Estimated cost of location update with polling de-registration scheme is given as follows:

$$
\begin{aligned}
M_{H L R-V L R \text { (quing }}= & {\left[P\left(m_{a, n^{-}}-1\right) \times C_{m}(1)+2 \times 1\right] } \\
& +\sum_{i=2}^{R} P\left(m_{a, n^{-}}-i\right) \\
& \times\left\{2 \times C_{m}(1)+C_{m}(R)+2 T(1, R)+2\right. \\
& \times 1\} \ldots .(4)
\end{aligned}
$$

The first part of this Eq. No.4 is the cost of location update in intra-VLR move. We have added 2 and its reason is explained previously. The second part shows the scenario after an interVLR move. T $(1, \mathrm{~L})$ is estimated in the same manner but this cost is multiplied by 2 because, we are not sending any deregistration request to the old VLR and no acknowled gement is being received in response. If MT resides in an RA for some times then MT will follow the polling de-registration scheme and hence $\mathrm{Cm}(1)+2 x 1$ is added.

Estimated cost of location update with timeout de-registration scheme is given as follows:

The first part of this Eq. No.5 is the cost of location update in intra-VLR move. We have added 1 and its reason is explained previously. The second part shows the scenario after an interVLR move. T $(1, \mathrm{~L})$ is estimated in the same manner but this cost is multiplied by 2 because, we are not sending any deregistration request to the old VLR and no acknowled gement is being received in response. If MT resides in an RA for some times then MT will follow the timeout de-registration scheme and hence $\mathrm{Cm}(1)+1$ is added.

$$
\begin{aligned}
M_{H L R-V L R \text { (tims out })} & =\left[P\left(m_{a, n^{\prime}}-1\right) \times C_{i m}(1)+1\right] \\
& +\sum_{i=2}^{R} P\left(m_{a, n^{-}}-i\right) \\
& \times\left\{2 \times C_{m_{i}}(1)+C_{m}(R)+2 T(1, R)\right. \\
& +1\} \ldots \ldots(5)
\end{aligned}
$$

Estimated cost of location update with group de-registration scheme is given as follows:

$$
\begin{aligned}
M_{H L R-V L R \text { (grovip) }}= & {\left[P\left(m_{a, n}-1\right) \times C_{m}(1)\right]+\sum_{i=2}^{R} P\left(m_{a, n}-i\right) } \\
& \times\left\{2 \times C_{m}(1)+3 \times C_{m}(R)\right. \\
& +2 T(1, R)\} \ldots \ldots \ldots(6)
\end{aligned}
$$

The first part of this Eq. No.6 is the cost of location update in intra-VLR move. When an MT leaves its RA and enters into new RA the new VLR sends a registration request to the HLR. HLR keeps the identification of the MT into the OML of the old VLR. After performing the MT's profile update by accessing its database HLR sends the acknowledgement message along with the OML of new VLR. We see that HLR database is being consulted three times. The first access is done for putting the MT's identification into the old VLR's OMT, second time for updating the MT's current location information and third time for emptying the OML of new VLR, further the entries of this OMT is sent back with the acknowledgement. At the VLR side database is being consulted twice, first for the registration of new MT and second for de-registration of the entries sent by the HLR.

\section{RESULTS}

In this section the numerical values of polling, timeout and group de-registration schemes with explicit HLR/VLR schemes are evaluated and compared. Fig 5 and 6 show the performance of location update schemes with $R=5$ and $R=3$ respectively. In the figures users are classified with respect to their moves. When $p$ (probability value) is very small, the mobile unit moves are not local. 


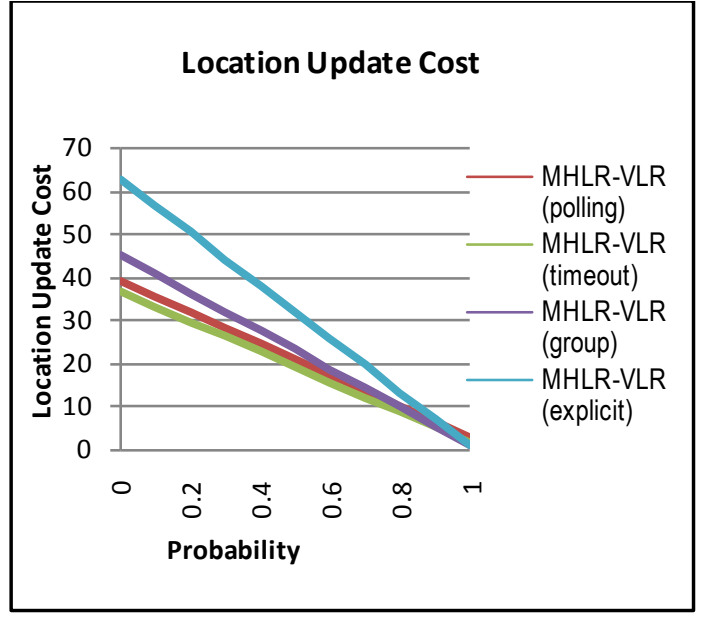

Fig. 5: Location update cost when $R=5$

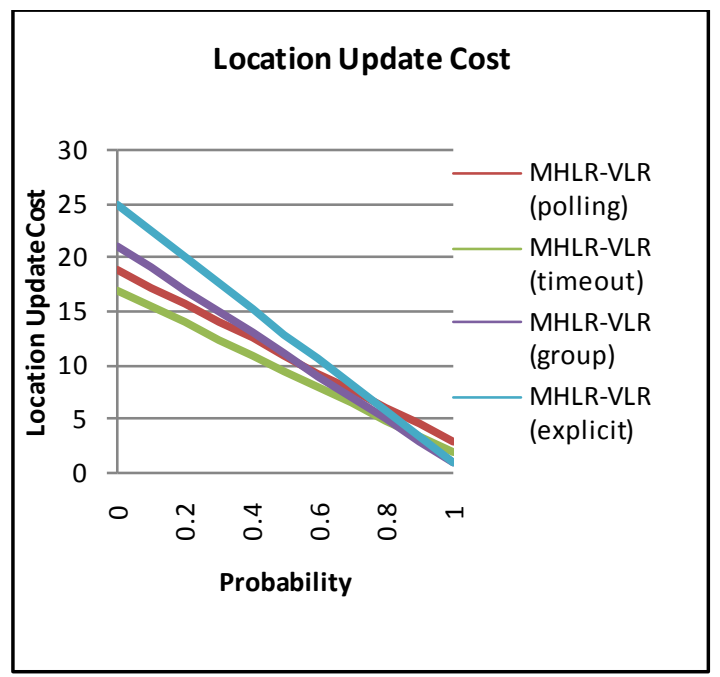

Fig 6: Location update cost when $R=3$

From fig 5 and 6 , it is clear that explicit de-registration scheme incurs more cost than the other three schemes. Among the three schemes, group de-registration scheme has the highest cost and timeout scheme has the lowest cost.

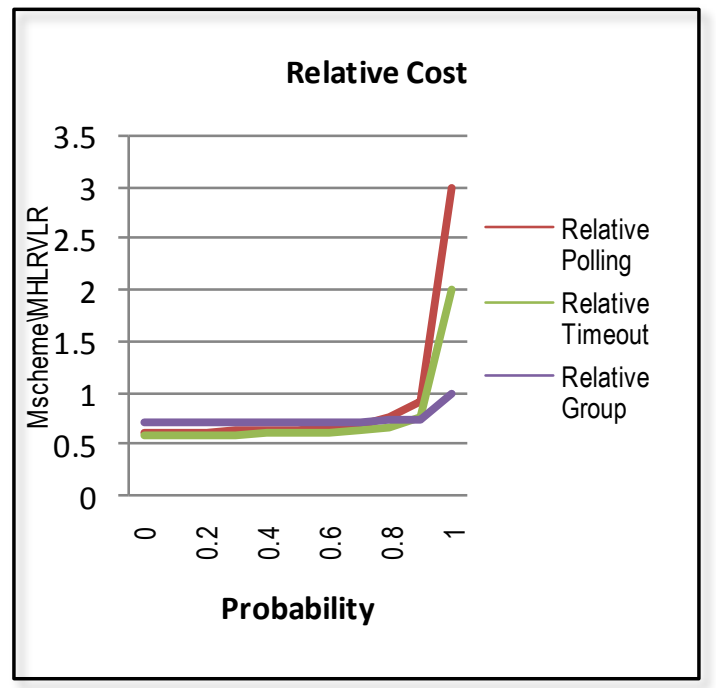

Fig 7: Relative location update cost when $R=5$
To measure the performance of the three de-registration schemes, relative costs of the schemes are evaluated against the explicit de-registration scheme. Fig $7 \& 8$ show the relative cost when $\mathrm{R}=5$ and 3 respectively.

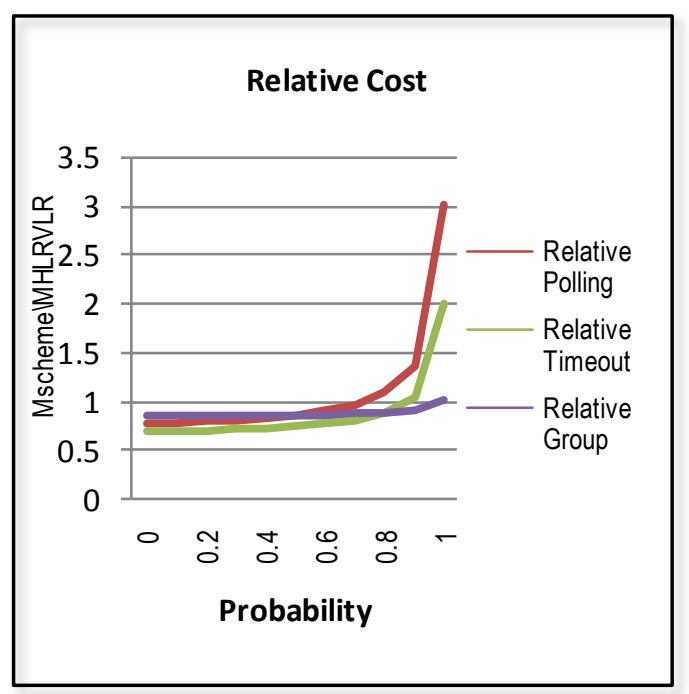

Fig 8: Relative location update cost when $R=3$

\section{CONCLUSION}

Analy sis done in the last section shows that total cost incurred into the group de-registration scheme is greater than polling and timeout scheme but less than the explicit de-registration scheme. It is to be noted that polling and timeout schemes help in removal of stale entries of the MTs after a fixed period of time from VLR. On the other hand the process of removing the stale entries from the VLR is dependent on the mobility not on time in group de-registration scheme and hence it is more efficient than the previous two. Finally we can conclude that group de-registration scheme is better than the other two. This analysis is made by taking a single HLR. Single HLR architecture incurs with the bottleneck problem. To solve the problem of bottleneck, multi-HLR architecture was proposed. In future group de-registration scheme can be used with the multi-HLR architecture.

\section{REFERENCES}

[1] I.F. Akyildiz and J.S.M. Ho, "On location management for personal communications networks",IEEE Communk. Mag., pp.138-145, Sept.1996.

[2] Zuji Mao and Chritos Douligeris, "Group De-registration Strategy for PCS Zuji Mao and Chritos Douligeris, "Group De-registration Strategy for PCS Networks," IEEE communications letters, vol.6, no.4, April, 2002.

[3] Vijay Kumar, Mobile Databases, JOHN WILEY \& SONS, INC., PUBLCATION.

[4] Y.-B. Lin, "De-registration strategies for PCS networks," IEEE Trans. Veh. Technol, vol.47,pp.49-57,Feb.1998.

[5] A.R. Noerpel, L.F. Chang and Y.-B Lin, "Polling de-registration for unlicensed PCS," IEEE J. Select. Areas Commun., vol.14, pp.728-734, May 1996.

[6] Rajeev R. Kumar Tripathi, Sudhir Agrawal, Swati Tiwari, "Modified HLR-VLR Location Management Scheme in PCS Network," IJCA (0975-8887), vol.6, no.5, Sept. 2010. 
[7] Y-B. Lin and A. Neorpel, "Implicit de-registration in PCS network," IEEE Trans. Veh. Technol., vol.43, no. 4. Pp. 10061010, 1994.

[8] EIA/TIA, "Cellular radio-telecommunications intersystem operations: Automatic roaming'Tech. report IS-41.3-B, EIA/TIA, 1991.

[9] M. Mouly and M-B. Pautet, "The GSM System for Mobile Communications,"Palaiseau, France, 1992.
[10] Ai-Chung Pang, Y-B Lin, Yuguang Fang "Implicit Deregistration with Forced Registration for PCS Mobility Management,"Wireless Networks 7, 99-104, 2001.

[11] Z. mao, C. Douligeris, "A novel deregistration strategy for mobile networks”, IEEE VTS 2750-2754, vol. 6, Aug 2002.

[12] A.R. Noerpel, Y.B. Lin and H. Sherry, "PACS: Personal Access Communication System- A Tutorial", IEEE Personal Communications, vol.3, no.3, pp.32-43,June 1997. 